Research Article

\title{
Geological Modeling Technology and Application Based on Seismic Interpretation Results under the Background of Artificial Intelligence
}

\author{
Ximing Peng, ${ }^{1,2}$ Minglu Li, ${ }^{3}$ Yalin $\mathrm{Zhu}^{1,4} \mathrm{Na} \mathrm{Li,}{ }^{5}$ and Hao Dong $\mathbb{C}^{6,7,8}$ \\ ${ }^{1}$ State Key Laboratory of Continental Dynamics, Department of Geology, Northwest University, Xi'an 710069, Shannxi, China \\ ${ }^{2}$ Institute of Geo-Environment Monitoring of Jingxian, Xuancheng 242500, Anhui, China \\ ${ }^{3}$ School of Media, Zhengzhou Institute of Engineering and Technology, Zhengzhou 450045, Henan, China \\ ${ }^{4}$ Henan Provincial Academy of Building Research, Zhengzhou 450053, Henan, China \\ ${ }^{5}$ School of Doctor of Business Administration, Jose Rizal University, Mandaluyong 1552, Philippines \\ ${ }^{6}$ Shaanxi Provincial Land Engineering Construction Group Co., Ltd, Xi'an 710075, Shannxi, China \\ ${ }^{7}$ Institute of Land Engineering and Technology, Shaanxi Provincial Land Engineering Construction Group Co. Ltd., Xi'an 710075, \\ Shannxi, China \\ ${ }^{8}$ School of Management, Xi'an Jiaotong University, Xi'an 710049, Shannxi, China
}

Correspondence should be addressed to Hao Dong; donghao19@stu.xjtu.edu.cn

Received 27 August 2021; Revised 5 October 2021; Accepted 12 October 2021; Published 5 November 2021

Academic Editor: Sang-Bing Tsai

Copyright ( 2021 Ximing Peng et al. This is an open access article distributed under the Creative Commons Attribution License, which permits unrestricted use, distribution, and reproduction in any medium, provided the original work is properly cited.

\begin{abstract}
The development of seismic technology has made seismic data to be widely used in the interpretation of stratigraphic sequence frames, reservoir identification, fluid detection, and other research fields involved in reservoir description. The 3D technology reservoirs have always been the focus, as well as difficulty, of research. With the rapid development of information technology and the continuous improvement of seismic exploration level, people have put forward higher requirements for the accuracy of seismic data interpretation results. Aiming at the large number of structural and unstructured data in seismic, logging, geology, and other disciplines involved in seismic interpretation, how to effectively organize and coordinate analysis to discover the hidden reservoir structure and oil and gas distribution information has always been a geological and important topic for information processing technicians. This thesis is aimed at the current high-water-phase development of Shengtuo Oilfield reservoir and the problems existing in geological research. Based on seismic structural interpretation and attribute analysis, this paper analyzes the reservoir structural characteristics, sedimentary characteristics, and reservoir physical parameter characteristics based on geology, logging interpretation, core analysis, drilling, and seismic interpretation. Using the kriging method with external drift can cooperate with seismic variables to establish a reservoir geological model to study the Shengtuo Oilfield reservoir. We combine artificial intelligence technology with geological modeling technology of seismic interpretation results to explore the best method for predicting earthquakes. The research results in this paper show that the relative error of the model established by the kriging method in the article is relatively small for thinning wells, mainly concentrated around 1\%. Examination of the thinning wells of 45 wells shows that the model established is basically good and the example has high accuracy. The research results in this paper have a guiding study of distribution and tapping potentials in the study area, formulating reasonable development and adjustment plans and improving oil recovery.
\end{abstract}

\section{Introduction}

With the development and application of database technology, large amounts of data are stored in databases or data warehouses. These data are often multidimensional. How to fully mine the useful information behind complex data has become the goal of people's research. Among the many problems that have emerged, the multidimensional and unstructured data are the most significant. Traditional methods are difficult to handle this change. The analysis and 
research of multidimensional data has emerged as the times require. Multidimensional analysis organizes the data in a multidimensional form, which can flexibly perform different analysis operations on the data and observe and analyze the data from different angles, so as to understand the information and connotation behind the complex data. The complex and multidimensional data structure promotes the wide application and rapid development of multidimensional data analysis technology. Growing technology has made seismic data widely used in multiple research fields involved in the interpretation of stratigraphic sequence frames, reservoir identification, and fluid detection; using seismic interpretation of isochronous tectonic plates and faults, combined with well contrast, stratification and stratigraphic development characteristics, the application of high-precision 3D area, and the improvement of seismic reservoir interpretation levels have made the reliability of seismic description reservoirs increasingly greater $[1,2]$. The difference between complex fault block reservoirs and conventional reservoirs is that reservoirs are often complicated by faults. Below these reservoirs, faults are an important part. As we all know, oil is one of the important energy sources. It affects global economic development, military security, and political stability and is a force that can influence the world situation. Therefore, petroleum exploration research has always been a subject of enthusiastic research. Seismic data is the main source of information for exploration research. The organic combination of information, especially the study of fully excavating the internal information of seismic data, has achieved many results and has improved and expanded the level of exploration. Its main features are all related to or controlled by faults, manifested in many faults at different levels, different directions, different periods, and different mechanical properties in the oilfield. The structure is cut and broken to form smaller fault blocks, forming fault block groups with different formation and genesis [3, 4], and the structural characteristics and other characteristics of such reservoirs. Reservoir geological modeling has a good condition. At present, the difficulty of oilfield development and production and development costs are rising. The comprehensive use of multidisciplinary information is of great significance. It can save exploration and development costs, reduce risks, and improve benefits. In the past, methods often encountered bottlenecks on specific problems and were difficult to be widely used. Therefore, the techniques and methods to fully utilize multisource information and explore the geological features contained in multidimensional information need further research. Petrel software is made of precious models, carrying out fine anatomy of the internal structure of the reservoir, revealing the distribution, and characterizing the geological characteristics of three-dimensional spatial distribution of various attributes that can provide accurate three-dimensional geological bodies for the accuracy of numerical simulations to study the distribution of remaining oil and make the description of reservoir heterogeneity more accurate, prejudice of making reasonable development plan and taking effective production measures [5-7]. The Shengtuo Oilfield reservoir is a rolling anticline reservoir complicated by faults. It is a sedimentary system. The geological structure is relatively complex, the sand body changes laterally rapidly, and the reservoir heterogeneity is strong. The fine geological research work of the Shengtuo Oilfield No. 1 area started late, and the basic work of reservoir geological research is very weak. Seismic and logging data are important data in oilfield geological research. In a broad sense, seismic interpretation can be regarded as the application of seismic data for exploration research, including structural interpretation, stratigraphic interpretation, lithology interpretation, hydrocarbon interpretation, and other issues. In recent years, with the development of petroleum geology, many new technologies and methods require the comprehensive use of multidisciplinary materials. The purpose of this article is to propose effective analysis methods for the multidimensional data involved in seismic oil and gas prediction and seismic and sonic logging matching process to improve the accuracy of prediction and matching. So far, no overall fine oil reservoir description work has been carried out, which has led to the stratigraphic framework and sedimentary characteristics. The understanding of structural characteristics, reservoir characteristics, and geological models is relatively vague, and it should not be necessary for the development and adjustment of reservoirs in the subsequent water flooding stage [8-10]. The development status of Shengtuo and the difficulties of geological research, the kriging modeling as a structural thing, is under the control of sedimentary microfacies. Improving the accuracy of oil and gas prediction can reduce exploration and development costs, reduce risks, and improve benefits. The effective matching of seismic logging data is a bridge between the two, and it is the basis and key issue of the exploration process such as reservoir description, reservoir inversion, and seismic lithology interpretation. Therefore, it is of great significance to study fine oil and gas prediction methods and seismic logging data correlation matching methods. The spatial physical properties were studied, the heterogeneity of the material was clarified, and the visualization of the oilfield was realized. This will further study the distribution of next reservoir development adjustment, and the schemes and further enhancement of recovery factor are of great significance [11, 12].When technology grows, smart devices will be able to enter different areas of society through a wide range of advanced technologies such as information security, smart manufacturing, smart medical care, transportation, intelligence, smart agriculture, information security, and machine manufacturing, enabling them to exert tremendous power and influence in society. Therefore, its role in the field of geology is also obvious. This subject is aimed at the research of information fusion and multidimensional data analysis based on the oil and gas prediction problems based on geophysical exploration data such as seismic and well logging. First, we perform the correlation analysis and processing between the seismic logging data, divide the two into a unit domain through the well-seismic matching method, and organize the multisource influencing factor data in the oil and gas prediction process with a multidimensional data model. Through analysis and processing, corresponding 
multidimensional analysis method is proposed, and the method of combining support vector machine and information fusion is used for oil and gas prediction [13, 14].

This chapter first analyzes the reasons and manifestations of the mismatch of seismic logging data and then introduces the process of well-seismic matching. The timedepth conversion, synthetic seismic record production, and matching algorithms in the process steps are studied separately, and the correlation matching is comprehensively obtained. These include establishing a velocitydepth model based on the time migration velocity and proposing an adaptive time-depth conversion, considering the influence of the actual density logging curve to obtain the reflection coefficient. Three-dimensional geological modeling is one of the key technologies for spatial study and visualization data. To enhance speed, efficiency, and accuracy of geological modeling, Tang et al. proposed a pointline-area-body fast progressive 3D technology method. Tang et al. first used drilling formation data to establish a geological profile through human-machine interaction and then restored the true location of the geological profile in threedimensional space based on the drilling coordinates. Based on this, Bingyin Tang established a series of stratigraphic models using Kriging interpolation using the same stratigraphic lines on the profile. On this basis, the establishment of the framework model is completed. Finally, Tang et al. used binary space segmentation (BSP) vector shearing technology to cut the model boundary and established 3D learning area $[15,16]$. In complex reverse fault block reservoirs, the same reservoir may be repeatedly drilled in vertical wells, making geological modeling of these reservoirs one of the most troublesome modeling activities. Traditional reservoir geological modeling methods cannot well describe the superimposed structure and reservoir characteristics of inverse fault block reservoirs, which leads to inaccurate and unreliable reservoir geological models, and deformation and reserve loss [17]. Due to the loss of reservoirs and reservoirs, actual reservoirs cannot be described using scale-up models. To this end, Renbo Song has developed a series of technologies to solve this technical problem, mainly including multiboundary technology, fault isolation technology, superimposed reservoir area full coverage rectangular corner point grid system, and other reservoir numerical simulation amplification technologies. We established an effective solution for structural modeling, numerical simulation upgrade, and other problems in the geological modeling of complex reverse fault block reservoirs. Song et al. comprehensively used seismic tectonic interpretation, sedimentary facies, stratigraphic contrast, wells, and other data to establish an accurate Yingdong 1 reservoir geological model and a dynamic prediction model of reservoir numerical simulation. The actual application shows that the matching rate between the predicted output and the 2-year actual output exceeds 95\% [18, 19]. The systems formed by different combinations of holes, caves, and fractures are the main research objects. Reservoir modeling is the key technology for reservoir description. The method of 3D geological modeling of carbonate reservoirs of great significance and strong anisotropy is different from that of clastic reservoirs. Guo et al. used geostatistical algorithms on the basis of accurate modeling of structures. Combining the advantages of the organic method with deterministic features, the main stress zone is described as a whole, and it is applied to the 3D technology carbonate reservoirs to match the integration of well-seismic data during the modeling process and form a comprehensive modeling method for carbonate reservoirs, with dynamic and static data constraints being provided to the accurate reservoir numerical, a high-precision combination of Middle Ordovician carbonate reservoirs for the exploration and development of Halatang Oilfield. The deployment of efficient well groups provides a reliable basis [7, 20]. With the increasing difficulty of ore prospecting in shallow surface deposits and the increase in the depth of resource exploration, the application of $3 \mathrm{D}$ modeling technology in deep ore prospecting becomes more and more obvious, and its accuracy directly determines the degree of understanding of the geological bodies and the metallogenic conditions. To this end, $\mathrm{Qu}$ et al. once proposed a set of anomalous information extraction technology combining aeromagnetic data processing and 3D geological modeling. Invert the selected ones to obtain the geological models of each section: the $3 \mathrm{D}$ geological unit by the section connection method. The 3D geological model of the undulating terrain is a model of every place $[21,22]$; reward simulation results of the entire 3D geological model and geological unit are presented. $\mathrm{Qu}$ et al. added a reasonable geological constraint and modified the model through a comparative analysis with the test results. Through multiple adjustments, the model was brought to the maximum extent close to the actual situation. The model can reflect the geological information well $[23,24]$.

This paper introduces the kriging method. After analyzing the structural characteristics, sedimentary appearance, or reservoir parameter Shengtuo Oilfield reservoir, the Kriging method with external drift, which can cooperate with seismic variables, made reservoir geological model to study the Shengtuo Oilfield reservoir. The relative error of the geological model established for thinning of this paper mainly concentrated around $1 \%$. It can be known that the established model is basically good and it has higher accuracy. The research results of this paper have a guiding function of study in remaining distribution of study place, tapping potentials, formulating reasonable development and adjustment plans, and improving recovery factors [25]

\section{Proposed Method}

2.1. Overview of Reservoir Geology and Development. Shengtuo Oilfield is located in Dongying Sag in the Jiyang Depression, with the Chenjiazhuang bulge in the north, Kendong Qingqingzi bulge in the east, Lijin oil sag in the southwest, and Minfeng oil sag in the east. The Dongxin oilfield in the central uplift belt is connected with a late Cretaceous, Tertiary fault, Yanqi complex basin surrounded by bulges. The general features are relatively simple base structure and complicated cover structure. There are few folds and many faults, and normal faults with different 
properties and different grades are basin-dipping fault systems. The Dongying Sag is located in the southern part of the Jiyang Depression in the Bohai Bay Basin. The sag is connected by adjacent faults to adjacent uplifts. Its basic structural type is a semi-earthed rift basin with a northern fault and a southern fault. The long-term inherited depressions are controlled by the surrounding bedrock bulges and large basin-dipping syngenetic fault zones, and traps formed by various faults become favorable locations. The oil and gas generated in the sag migrated to these traps and accumulated into it. It is in this geological background that Shengtuo Oilfield has become an inside place. Shengtuo Oilfield has a high-quality oil production area and a rich source supply area, as well as good oil and gas closed conditions. It is an oil-bearing area with abundant oil sources and provenances in Shengli Oilfield.

2.1.1. Structural Characteristics. The Shengtuo Oilfield formed with the Shenglicun anticline in the east and the Xinzhuang anticline in the west as the descending plate of the Shengbei fault. The tectonic location is located in the northern steep slope of Dongying Sag, Xinzhuang, Shengli Village. The northern part of the Yonganzhen fault zone is connected with the Chennan fault to the Chenjiazhuang bulge in the north, the Yonganzhen fault zone in the east, the Lijin fault zone in the west, and the central fault zone in the south. The Dongying structure is connected by a saddle and is connected to the Lijin deep depression in the southwest. It is a reverse traction anticline structure on the descending plate of the Shengbei arc-shaped large fault. The main structural system in the Shengtuo area is composed of the northern steep slope fault system and the central anticline structural belt, which can be further divided into three secondary structural units, namely, the northern two steps, the central anticline structural belt, and the nasal structural belt, southern depression zone.

2.1.2. Stratigraphic Characteristics. Drilling data reveals that the Paleogene and Neogene strata in Shengyi District are in order of Kongdian Place, Shahejie Formation (divided into Sha 4, Sha 3, Sha 2, and Sha 1), Dongying Formation, and Guan Tao Formation and Minghua Town Formation. It is one of Shahejie Formations as the main oil-bearing formation. From the Paleogene Shahejie Formation to the Neogene Guantao, Minghuazhen Formation, the Shengtuo Oilfield has undergone two large sedimentary cycles. From the third part of Sha 3 to the upper part of Sha 2 is a process from deeper lacustrine to delta. The river facies: the top of Sha 2 to Sha 1, Dongying Formation, Guantao. The Minghuazhen Formation again entered from water receding, sedimentary evolution from lakes to deltas, rivers.

\subsubsection{Reservoir Characteristics}

(1) Rock Mineral. The composition of the oil layer is on the bottom. The upper part of the sandstone is sorted, and the bottom part is poorly selected. The cement types are mainly contact and pore contact. The cements are clay minerals of montmorillonite and kaolinite.

(2) Lithological Characteristics. The 1.3 sand layer group in the second member of Shahejie Formation is composed of purple-red, gray-green mudstone, fine material. The sandstone thickness is $2.4 \mathrm{~m}$, and the maximum thickness is $10 \mathrm{~m}$.

(3) Sand Formation Group. Sand bodies are mostly positive rhythms or composite rhythms. The lithology of the lower sand group is coarser and thinner upward, and the sand group is coarser and finer at the granularity.

(4) Reservoir Parameter Distribution Characteristics. Sedimentary microfacies control the morphology and distribution of sand bodies. Sand bodies in small layers are mainly distributed in a network and band shape. The thickness of sand bodies in the central phase is relatively large, and the sand bodies in the edges are thin. High-porosity and highpermeability reservoirs are mostly distributed in places where sand stones are developed, sand bodies are not developed, or the porosity and permeability of thin sands are significantly worse.

\subsubsection{Formation Temperature and Pressure Characteristics.} The original got 20.2 points, saturation pressure got 10.9-14.2 $\mathrm{MPa}$, and the ground-saturated pressure difference was 6-9 $\mathrm{MPa}$. The original formation temperature was $80^{\circ} \mathrm{C}$, and after years of waterflooding development, the current formation temperature has dropped to $750^{\circ} \mathrm{C}$.

2.2. Deterministic Modeling Methods. At present, a combination of deterministic modeling and stochastic modeling is generally used to establish reservoir geological models. Deterministic modeling is based on deterministic data and inferring unique and definite reservoir parameters between points. However, in the case of imperfect data, there are still some uncertain factors in people's understanding of the reservoir, and it is difficult to grasp its true characteristics. Therefore, the reservoir is considered to be random. Stochastic modeling is based on known information, using random functions as a theory and applying random simulation methods. This method recognizes that reservoir parameters outside the control point have a certain degree of uncertainty, that is, a certain degree of randomness. Big data technology is the product of the development of a specific level of network under the background of artificial intelligence. It helps people solve practical problems, so it is important not only to create a good network environment, but also to improve the practicality and flexibility of the network. It can be seen that big data technology has a lasting impact on the development of the future society.

2.2.1. Reservoir Seismic Method. Reservoir seismology methods mainly use seismic data to study the geometry, lithology, and parameter distribution of the reservoir. Starting from known well points, applying seismic lateral prediction technology to predict interwell parameters and 
establishing a 3D touch machine. This method mainly includes 3D seismic and cross-well-seismic methods.

2.2.2. Reservoir Sedimentology Method. Reservoir sedimentology method is based on isochronous stratigraphic contrast and sedimentary model and establishes the reservoir structure model through the cross-well sand body contrast. The interwell comparison of model and single-well facies analysis: generally, the interwell interpretation relies on similarity or difference of the cross-well log curves. A more scientific method uses multiple disciplinary method for comprehensive and integrated interpretation.

2.2.3. Kriging Method. Interwell interpolation is a common method for establishing a deterministic reservoir parameter distribution model. Based on some known information, a variation function is used to make an optimal and unbiased estimate of the unknown value of the estimated point. The kriging method minimizes the variance of the error. The ordinary kriging method is a more commonly used method. The basic principle is as follows.

It is assumed that the regionalized variable $Z(x)$ satisfies the second-order stationary hypothesis and eigenhypothesis. Its mathematical expectation is $m$, the covariance function is $c(h)$, and the mutation function $r(h)$ exists, which is

$$
\begin{aligned}
E[Z(x)] & =m, \\
c(h) & =E[Z(x) Z(x+h)]-m^{2}, \\
r(h) & =\frac{1}{2} E[Z(x)-Z(x+h)]^{2} .
\end{aligned}
$$

Let $Z(x)$ be a second-order stationary random function, which samples at $n$ locations, $Z\left(x_{1}\right), Z\left(x_{2}\right), \ldots, Z\left(x_{n}\right)$, and the estimated amount at point $x_{0}$ is

$$
Z *\left(x_{0}\right)=\sum_{i=1}^{n} \lambda_{i} Z\left(x_{i}\right)
$$

Among them, $\lambda_{i}$ is the weight coefficient, which indicates the degree of contribution of the observation $Z\left(x_{i}\right)$ to the estimated value $Z *\left(x_{0}\right)$ at each spatial sample $x_{i}$. The key of the kriging algorithm is to calculate the weight coefficient $\lambda_{i}$. The calculation of the weight coefficient must meet two conditions:

(1) Let $Z *(x)$ is an aim of $Z\left(x_{i}\right)$, namely, $E[Z *(x)]=E[Z(x)]$. At the time $E[Z *(x)]=m$, namely,

$$
E\left[\sum_{i=1}^{n} \lambda_{i} Z(x)\right]=\sum_{i=1}^{n} \lambda_{i} E\left[Z\left(x_{i}\right)\right]=m .
$$

There is $\sum_{i=1}^{n} \lambda_{i}=1$.

(2) Minimize the estimated variance; that is, minimize the number of value $Z *(x)$ or number of value $Z\left(x_{i}\right)$. It can be expressed by the variance function:

$$
\sigma^{2}=c(x, x)+\sum_{i=1}^{n} \sum_{j=1}^{n} \lambda_{i} \lambda_{j} c\left(x_{i}, x_{j}\right)-2 \sum_{i=1}^{n} \lambda_{i} c\left(x_{i}, x_{j}\right) .
$$

To minimize the estimated variance, according to the Lagrangian multiplier principle, let $F=\sigma^{2}-2 \mu\left(\sum_{=1}^{n} \lambda_{i}-1\right)$, find the partial derivative of $F$ to $\lambda$ and $\mu$, and let the partial derivative be 0 to get kriging:

$$
\left\{\begin{array}{l}
\frac{\partial F}{\partial \lambda_{i}}=2 \sum \lambda_{i} c\left(x_{i}, x_{j}\right)-2 c\left(x_{i}, x_{j}\right)-2 \mu=0 \\
\frac{\partial F}{\partial \mu}=-2\left(\sum_{i=1}^{n} \lambda-1\right)=0(i=1,2, \ldots, n)
\end{array}\right.
$$

After finishing,

$$
\left\{\sum_{j=1}^{n} \lambda_{j} c\left(x_{i}, x_{j}\right)-\mu=c\left(x_{i}, x_{j}\right) \sum_{i=1}^{n} \lambda_{i}=1(i=1,2, \ldots, n) .\right.
$$

Solve system of linear cases (8), find the weight coefficients and Lagrangian multipliers, bring them into formulae (4) and (6), and get the estimated value and estimated variance, respectively. In the presence of the variogram, according to the covariance function and the relationship of the variogram, $c(h)=c(0)-\gamma(h)$, and the variogram is used to represent, that is,

$$
\left\{\sum_{j=1}^{n} \lambda_{j} \gamma\left(x_{i}, x_{j}\right)+\mu=\gamma\left(x_{i}, x_{j}\right) \sum_{i=1}^{n} \lambda_{i}=1(i=1,2, \ldots, n)\right.
$$

A system of examples can also be represented by a matrix.

$$
\begin{aligned}
K & =\left[\begin{array}{ccccc}
C_{11} & C_{12} & \ldots & C_{1 n} & 1 \\
C_{21} & C_{22} & \ldots & C_{2 n} & 1 \\
\vdots & \vdots & \vdots & \vdots & \vdots \\
C_{n 1} & C_{n 2} & \ldots & C_{n n} & 1 \\
1 & 1 & \ldots & 1 & 0
\end{array}\right], \\
\lambda & =\left[\begin{array}{c}
\lambda_{1} \\
\lambda_{2} \\
\vdots \\
\lambda_{n} \\
-\mu
\end{array}\right], \\
D & =\left[\begin{array}{c}
c\left(x_{1}, x\right) \\
c\left(x_{2}, x\right) \\
\vdots \\
c\left(x_{n}, x\right) \\
1
\end{array}\right] .
\end{aligned}
$$

The ordinary kriging equations are 


$$
K \lambda=D .
$$

Solving equation (11), we can get $\lambda=K^{-1} D$, and its estimated variance is

$$
\sigma^{2}=c(x, x)-\lambda^{T} D .
$$

The premise of ordinary kriging interpolation is as follows: according to the structure of the space field, select an appropriate variogram model and find the variogram: (1) gridding, that is, a range of selected area and the size of the grid; (2) calculating the coordinates of the estimated point (that is, the grid node); (3) according to the search strategy (close-point distance search, orientation search), select the appropriate reference point; (4) find the coefficients of the system of equations based on the mutated function that has been obtained; (5) solve the system of equations (using the LU method) and find the weight coefficient $\lambda_{i}$; (6) the estimated point in formula (4) nis the number of interpolation points; (7) repeat steps (2) to (6) until the values of the grid nodes are all obtained.

\subsection{Stochastic Modeling Method}

(1) Three-dimensional geological modeling of commonly used stochastic models is the core content of geological research in the reservoir development stage, a summary of the foregoing basic research work, and the basis of numerical simulation. According to the random characteristics of research phenomena, random models can be divided into discrete models, continuous models, and hybrid models; according to the characteristics of the simulation unit, the random models can be divided into target-based random models and pixel-based random models as follows:

(i) Discrete model (based on target): this trick is rock type distribution, fracture and fault distribution, size, etc. Punctuation, truncated Gaussian random fields, Markov random fields, and two-point histograms are discrete random models.

(ii) Continuous model (based on pixels): this equation is used to describe the characteristics of continuous changes of reservoir parameters: the spatial distribution of porosity, permeability, and fluid saturation. The Gaussian domain and the fractal random domain are continuous models.

(iii) Hybrid model (two-step model): in practical applications, the above two types of models are often combined together. The first step is to establish a discrete model to describe a wide range of heterogeneous characteristics of the reservoir. The second step is to establish a continuous model to represent the spatial variation and distribution of rock parameters.

(2) Applicability about the stochastic model.
Since the application of stochastic simulation in the petroleum field, a variety of stochastic simulation methods have been developed based on different purposes, including different stochastic models and different simulation algorithms, such as Boolean models, truncated Gaussian domains, indicator simulations, Markov random domains, and twopoint and multipoint histograms, and have different simulation effects due to different simulation methods. Therefore, appropriate random models and different simulation algorithms should be selected according to the research object. Many scholars at home and abroad have conducted research and obtained very effective.

(3) Sequential Gaussian simulation method.

The sequential Gaussian simulation method is fast and simple. It is more suitable for simulating some rock physical properties with continuous middle values and scattered ends, such as porosity. The principle is as follows:

Let $Z_{i}$ be $N$ random variables. Based on the calculation of the variogram, the ordinary kriging equations are solved to obtain the mean and variance of the regionalized variable distribution to determine the cumulative conditional distribution function (ccdf) given any type of $n$ conditional data. Take a sample from (ccdf) and participate in the next simulation. Random variables are required to follow a normal distribution.

(4) Sequential Gaussian cosimulation method.

Sequential Gaussian cosimulation is to find the cumulative distribution function of each node along different random paths and extract the simulation value from the cumulative distribution function. This method can realize the constraint between wells and realize the lateral prediction of the reservoir. The purpose is to restore the multivariable spatial relationship. Because of the interdependent relationship between reservoir physical parameters, the simulation of collaborative regionalization will certainly be more consistent with the geological situation.

2.4. Model Construction. The model is composed of stratigraphic models and fault models. It is an important basis for $3 \mathrm{D}$ geological modeling. It directly reflects the 3D spatial framework of the reservoir. It is also a comprehensive reflection of the structural features and small layer features of the geological body built. Therefore, the accuracy of its establishment directly affects the accuracy of the later reservoir parameter model.

2.4.1. Stratigraphic Model. In order to control the reservoir characteristics and distribution law of the sand body deposition unit, the stratigraphic model must be refined, and the control interface of the stratigraphic model must be refined to a small level, establishing the layer model of each 
layer. The geological stratified data is used as the condition data, so that each small layer model built reflects the structural fluctuations of the stratum more accurately. However, due to the imperfection of basic data and the complexity of geological conditions, there are often many anomalous points in the calculation results of the software that are not in harmony with the surroundings. The solution is based on the implementation of basic data; combined with geological knowledge, it is corrected. The method of "topbottom-bottom, then-insertion" is used to better solve the relationship between layers and thickness.

2.4.2. Fault Model. There are many methods for modeling faults, and basically, they simulate the production of faults based on different fault data (such as the depth of breakpoints encountered by single wells, fault lines obtained from seismic tectonic interpretations, fault polygons, and stratigraphic gaps) shape (trend, tendency, inclination, curvature, etc.). But the choice of the final method depends on the data of the fault modeling. The ultimate goal of fault modeling is to establish a set of fault columns that reflect the properties of the fault plane (inclination, azimuth, spatial extension, curvature, etc.). The fault distance is controlled by the structural drop (hierarchical data). For places where the fault distance does not match the geological understanding, it is controlled by adjusting the control point of the intersection of the fault and the plane.

\section{Experiments}

3.1. Modeling Steps. The Petrel software is a complex fault block reservoir using the kriging method, and a structural model of the reservoir (including stratigraphic model and fault model), a sedimentary microfacies model, and an attribute model are sequentially established:

(1) Collect various types of data including drilling data, small layer and sand body data, fault data, and other related data, and build a layer model of the reservoir on this basis.

(2) Establish a reservoir fault model. The fault model is the basis of the geological model of complex fault block reservoirs. Only by establishing a high-accuracy fault model can the facies model and attribute model be established on this basis. In the simulation of faults, the faults and the top-bottom surfaces of the three-dimensional seismic interpretation should be used to construct the fault model based on the combination of fault points obtained from drilling.

(3) After the establishment of the layer model and the fault model, the structural model of the reservoir is obtained. 3D sedimentary microfacies are always used. Different sedimentary microfacies control the physical properties of different sedimentary rocks; that is, the spatial distribution of them determines the distribution of rock physical properties. Therefore, the establishment of sedimentary microfacies models is the basis of phased modeling.
(4) Modeling of attribute is of good use. The attribute parameter model (porosity, permeability, and oil saturation) is based on the average and mean square error of various physical property parameters and is established on the basis of the sedimentary microfacies model skeleton.

3.2. Data Preparation and Preprocessing. Data preparation reservoir geological modeling must have a complete geological database using descriptions. To make the structural model, well data must be corrected for core elevation before it can be used for reservoir geological modeling, and faults must be located in conjunction with the breakpoint data encountered by the well. For deviated wells with large-angle well deflections, the well deflection must be corrected before modeling. According to the requirements of the modeling software Petrel, combined with the geological characteristics of the 1.3 sand layer reservoirs in the second member of Shahejie Formation in Shengyi District, this model was mainly based on the following data:

(1) Drilling data: including raw data and results obtained through drilling, such as wellhead data including well name, well position coordinate data, surface core elevation, well trajectory data (sounding, well deflection and azimuth), Buxin elevation data, and drill horizon data.

(2) Small layer and sand body data: including small layer and sand body top and bottom data divided by fine contrast.

(3) Fault data: fault data can be obtained from seismic interpretation or digitally from structural maps. The latter is used for this modeling, and there is also breakpoint data obtained from drilling.

(4) Logging data: including the original logging curve data, porosity, permeability, oil saturation, and permeability variation coefficient obtained from logging.

3.3. Well Deviation and Core Elevation Correction. It is generally believed that the well angle is less than 5 . The well is straight if the inclination is greater than 5 . However, it cannot reflect the vertical depth of each depth point of the well and the real bottom data of the structural item, and it must be corrected for the well deviation. Import the well deviation data into petrel software, and the software will automatically perform well deviation correction and generate well trajectory. Ground surface fluctuations and differences in core height have certain marketing for the establishment of the structural model. Therefore, the core compensation altitude correction should be performed before modeling. The altitude correction generally uses the sea level as the base model range and the grid determination. The grid needs to be designed during the modeling process. The grid shape, size, and direction of the geological model will affect the generation of sand body skeleton model and attribute model. Therefore, to establish an accurate 
sedimentary microfacies model and attribute model, a reasonable grid must be designed. When designing the grid of the 1.3 sand layer group in Shengyi District, the following factors were considered:

(1) The direction of the sediment source: the grid design should consider the main direction of the sediment source, which is conducive to data analysis and estimation of the variogram.

(2) The fluid supply direction in the production process: the grid direction should be as parallel as possible to the flow direction. This is a requirement of the reservoir numerical simulation seepage model. In addition, the grid direction is unified with the production well pattern, which can reduce the dead knot in numerical simulation points.

(3) The type and size of the grid, which simulates complex structural forms, are the most obvious advantages of the corner grid. If faults in the study area develop, this type of grid can be selected; the choice of grid size should not only meet the needs of small-scale random sand body simulation, but also fully reflect the accuracy of the structural horizon, because the reservoirs of the 1-3 sand formations' spacing is close to $250 \mathrm{~m}$. Considering the factors of development well pattern and faults, this study set the grid step size on the plane to $30 \mathrm{~m} \times 30 \mathrm{~m}$, and the plane grid number is $221 \times 141$. The vertical grid mainly considers the thickness of the interlayer. The final vertical grid number is 79 , and the total grid number is 2461719 .

\section{Discussion}

4.1. Porosity Simulation Analysis. The rapid development of information technology has brought us into an era of information data explosion. The vast and diverse information data is an important carrier of knowledge. In order to discover more useful knowledge from a large amount of complex information data, it is necessary for us to study information. The current reality data has the characteristics of being massive and multidimensional, and the data collection has also undergone significant changes. Its main characteristics mainly include having large data volume, being multidimensional, and being unstructured.

According to the reservoir parameters explained by the loaded logs, the porosity simulation of each small layer was analyzed and fitted. Table 1 and Figure 1 show the simulation analysis of porosity of each small layer of the 1.3 sand layer group.

From Table 1 and Figure 1, it can be seen that the minimum variation of the porosity simulation variation function of each small layer of the 1st sand group is 1310.7, the maximum value is 1443.6 , the minimum value of the secondary range is 689.3 , and the maximum value is 756.6 . The minimum value of the primary range of the sand group is 1411.5 , the maximum value is 1450.2 , the minimum value of the secondary range is 673.2 , and the maximum value is 714.9. The vertical range is 40.5 , and the nugget constant is 0 .
4.2. Transmittance Simulation Analysis. According to the reservoir parameters explained by the loaded log, the simulation analysis was performed. Table 2 and Figure 2 show the simulation analysis of the 1.3 sand layer group in the second member of Shahejie Formation in Shengyi District.

Among the many problems faced by data analysis, the multidimensional and unstructured data is the most significant. The previous method of unit function variables and linear analysis methods are difficult to handle this change. The usual method is to transform the unstructured information into a structured structure. Perform the analysis, but this change is often at the cost of losing part of the relevant information. The production of multidimensional data analysis technology effectively solves this problem. It proposes new analysis methods for complex multidimensional data and improves the utilization of data. It can be seen in Table 2 and Figure 2 that the minimum variation of the simulated variation function of the permeability of the small layers of the 1 sand layer group is $1434.6, \max$ is 1692.9 , $\max$ of the secondary range is 546.7 , and max is $875.8: 2$. The minimum value of the primary range of the layer group is 1356.9 , $\max$ is 1536.9 , min of the secondary range is 744.9 , and $\max$ is $784.2 ; 3$ min of the primary range of the sand group is 1239 , $\max$ is 1769.3 , and min of the secondary range is 682 , and max is 827.7 . The vertical range is 43.5 , and the nugget constant is 0 .

4.3. Analysis of the Prosodic Porosity Model. The prosodic layer porosity model test is performed on the model established by the method used, and the analysis results of the prosodic layer porosity model test result are shown in Figure 3.

A model is an expression of people's understanding and understanding of the system, process, thing, or concept under study. It is a formal description of the objective world by people. Different levels of abstraction in the real world are manifested as different levels of abstraction in the data model. For increasingly complex data information, the relational data model is no longer applicable in representing the structure and semantics of the data information, and the multidimensional data model has entered people's sight.

It can be seen from Figure 3 that after the prosodic layer porosity model is tested, the original porosity and simulated porosity of the thinning well are approximately equal and are distributed on a straight line of $Y=X$, indicating the establishment of actual geology. The case is basically consistent, indicating that the modeling method used in this paper is feasible.

4.4. Relative Error Analysis of Thinning Wells. Regarding the examination of the thinning wells on the model established by the method used, the relative error analysis results of the thinning wells are shown in Figure 4.

Aggregated data refers to the data set obtained by completing the aggregation of basic data through statistics or analysis, so this type of database is also called a statistical database. The data items stored in this type of database are different from ordinary databases, and the storage is no longer 
TABLe 1: Parameters of the variation function of the porosity simulation in the Erqi Oilfield.

\begin{tabular}{|c|c|c|c|c|c|}
\hline Arrangement & Main transformer $(\mathrm{m})$ & Subvariable range $(\mathrm{m})$ & Vertical range $(\mathrm{m})$ & Nugget constant & Abutment value \\
\hline $1(1-1)$ & 1443.6 & 753.6 & 40.5 & 0 & 0.76 \\
\hline $1(1-2)$ & 1310.7 & 690.3 & 40.5 & 0 & 0.88 \\
\hline $1(1-3)$ & 1328.4 & 725.1 & 40.5 & 0 & 0.84 \\
\hline $1(2)$ & 1440.5 & 689.3 & 40.5 & 0 & 0.8 \\
\hline $1(3)$ & 1423.2 & 714.5 & 40.5 & 0 & 0.68 \\
\hline $1(4)$ & 1397.8 & 724.6 & 40.5 & 0 & 0.8 \\
\hline $2(1)$ & 1450.2 & 729.5 & 40.5 & 0 & 0.64 \\
\hline $2(2)$ & 1421.3 & 673.2 & 40.5 & 0 & 0.76 \\
\hline $2(3)$ & 1411.5 & 732.1 & 40.5 & 0 & 0.8 \\
\hline $3(1)$ & 1498.7 & 703.5 & 40.5 & 0 & 0.96 \\
\hline $3(2)$ & 1429.6 & 714.9 & 40.5 & 0 & 0.82 \\
\hline $3(3)$ & 1435.9 & 698.5 & 40.5 & 0 & 0.75 \\
\hline
\end{tabular}

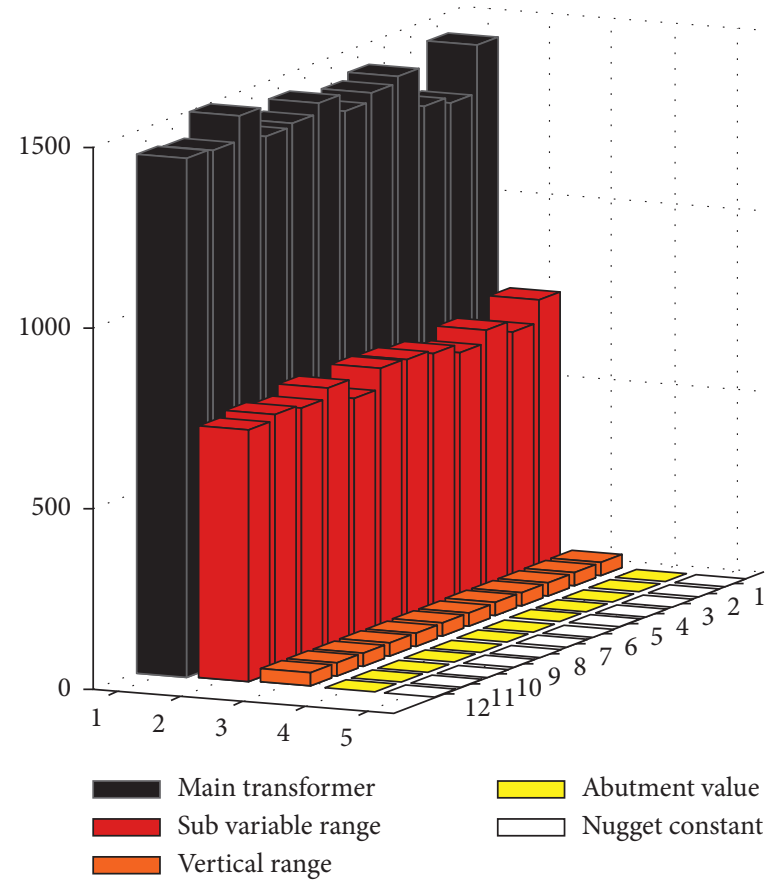

Figure 1: Simulation analysis of porosity of 1.3 sand formations in the second member of Shahejie Formation in the first block of the Erqi Oilfield.

TABLE 2: Variation function parameter table of permeability simulation of sand group 1-3 of Pu Oilfield.

\begin{tabular}{lccccc}
\hline Arrangement & Main transformer $(\mathrm{m})$ & Subvariable range $(\mathrm{m})$ & Vertical range $(\mathrm{m})$ & Nugget constant & Abutment value \\
\hline $1(1-1)$ & 1566.9 & 737.1 & 43 & 0 & 0.96 \\
$1(1-2)$ & 1692.9 & 776.1 & 43 & 0 & 0.88 \\
$1(1-3)$ & 1588 & 546.7 & 43 & 0 & 0.84 \\
$1(2)$ & 1754 & 875.8 & 43 & 0 & 0.9 \\
$1(3)$ & 1434.6 & 610 & 43 & 0 & 0.84 \\
$1(4)$ & 1497.8 & 674.6 & 43 & 0 & 0.88 \\
$2(1)$ & 1473 & 744.9 & 43 & 0 & 0.96 \\
$2(2)$ & 1536.9 & 773.7 & 43 & 0 & 1 \\
$2(3)$ & 1356 & 784.2 & 43 & 0 & 1.04 \\
$3(1)$ & 1609.8 & 827.7 & 43 & 0 & 0.96 \\
$3(2)$ & 1239 & 736.9 & 43 & & 0 \\
$3(3)$ & 1769.3 & 682 & 43 & & 0.85
\end{tabular}

simple data values, but relatively complex data structures, such as vectors, relations, and time series. Such data items are called statistical objects. Figure 4 shows that the relative thinning wells are relatively small, mainly concentrated around 1\%. Examination of the thinning wells of 45 wells shows that the model established has basically gotten accuracy. 


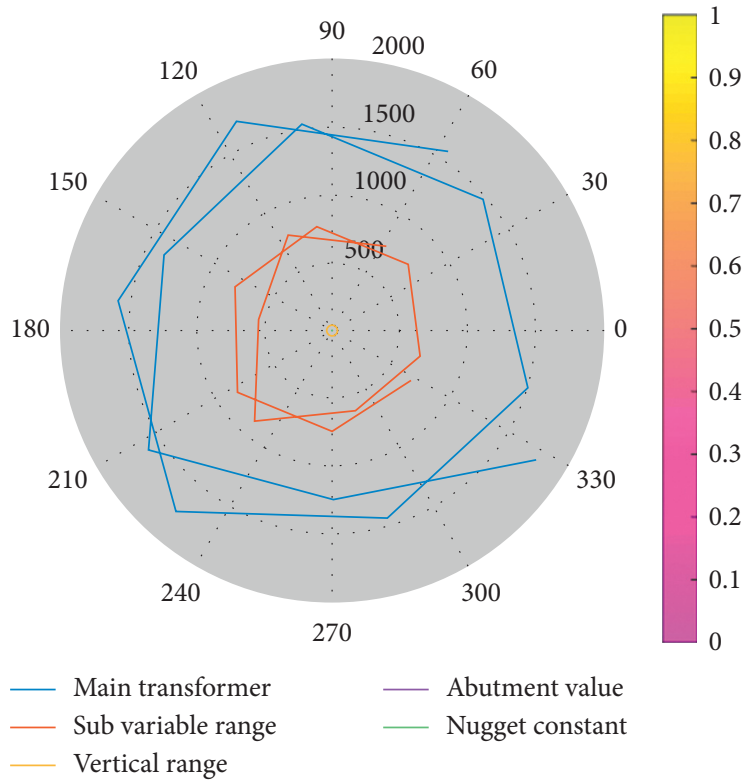

Figure 2: Simulation analysis of the permeability of the 1-3 sand formation in the second member of the Shahejie Formation in the first block of the Yanqi Oilfield.

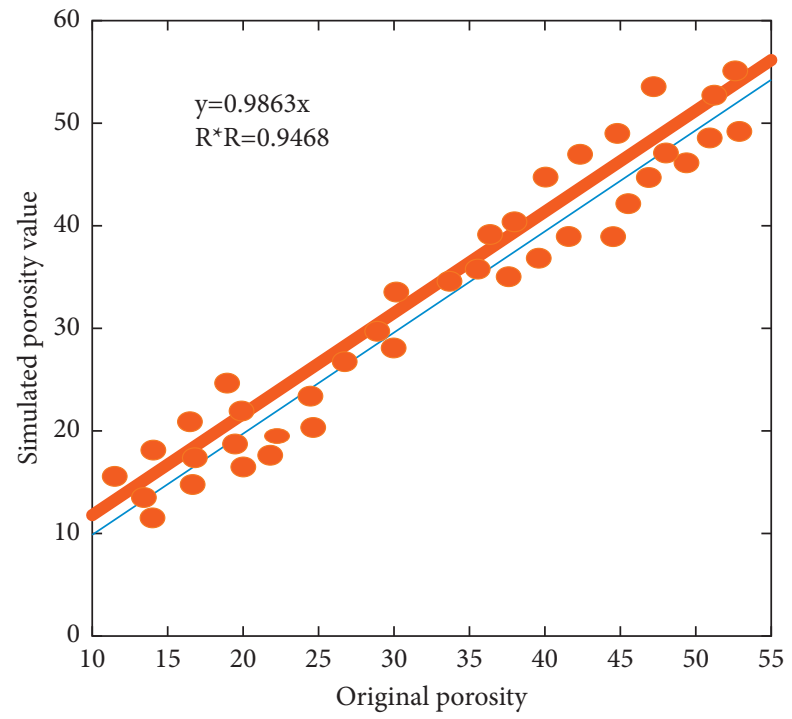

Figure 3: Analysis of the porosity model of thin layer pumping well.

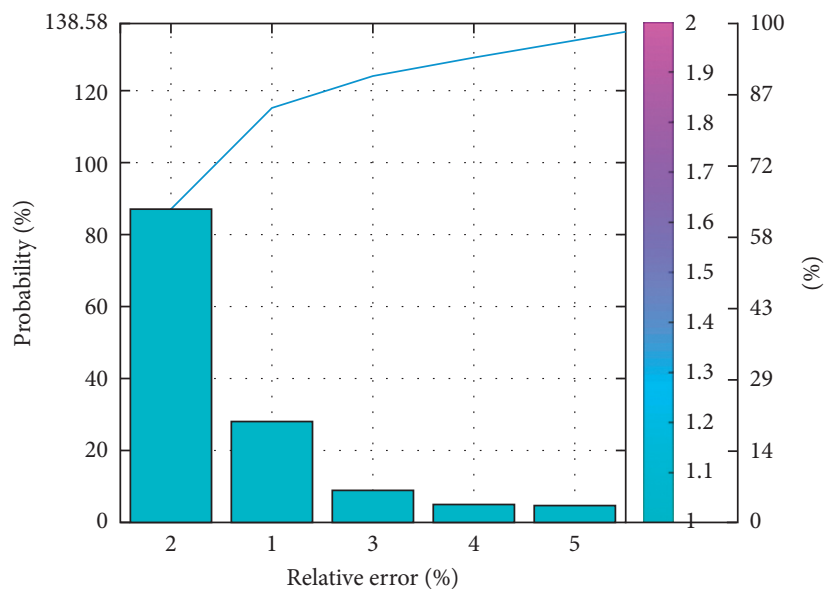

FIGURE 4: Analysis of thinning well inspection error. 


\section{Conclusions}

Information fusion was first called data fusion, which originated from early military applications. With the development of information technology, a more generalized concept of "information fusion" was proposed. Information fusion is inspired by the human brain's processing of complex problems: the processes of analyzing, predicting, and evaluating multisource information. Advances in seismic prediction technology have brought many benefits to people. Not only can it help people reduce casualties and property damage in earthquakes, but they can also play a guiding role in geological modeling technology. This thesis addresses the high water cut in Shengtuo Oilfield reservoir and the current development status and problems in geological research. Based on seismic tectonic interpretation and attribute analysis, based on geological, logging interpretation, core analysis, drilling, seismic interpretation, and other data, the structural characteristics, sedimentary characteristics, and storage of the reservoir are analyzed. The physical parameters of the formation are studied. The kriging method with external drift is capable of cooperating with seismic variables reservoir to study Shengtuo Oilfield reservoir. Analyzing the development process of artificial intelligence before and after, we can see that artificial intelligence often leads to failure when the culture of artificial intelligence changes in the environment, and the information does not match. So, the best way is to combine the external information community and social media to bring a huge impetus to the transformation of artificial intelligence. Seismic data and logging data are different manifestations of underground geology. Compared with the same reservoir petrophysical model, they have certain correlation characteristics. The combination of the two data can better perform seismic interpretation. However, because the seismic and logging methods are not used, they represent information of different scales. Therefore, there are certain differences between the two types of data and cannot be directly used in a comprehensive manner. The matching problem between the two has become a hot research topic.

The matching study of seismic data and logging data has become a major issue in the process of seismic lithology interpretation, reservoir inversion and detection, and reservoir description. For the problem of well-seismic matching, the logging data and seismic data are usually correlated by making synthetic seismic records. Based on actual seismic data, the logging data and seismic wavelet convolution operations are used to obtain synthetic seismic records to match and adjust. Make it match the seismic data next to the well accurately. Regarding the non-phase-controlled porosity and permeability model established by the method used in this paper, the property distribution is quite different from that in some areas of the sedimentary microfacies; and through phase-controlled attribute modeling, the distribution of reservoir properties and corresponding sand can be achieved. The consistency of the sedimentary microfacies makes the description of reservoir properties more accurate and reasonable. Therefore, the phased attribute modeling is more reasonable. It can be seen from the inspection of the simulated values and the coarsened values at the well points and the thinning well inspection that although there is a certain error between the simulated data and the actual geological conditions, this situation is unavoidable, and the error is within the allowable range. The model established has high accuracy, and the model is geological conditions, so the modeling method was considered feasible.

The seismic exploration method is to artificially select shot points on the ground in a specific area to excite seismic waves and obtain the response records of seismic waves to the elasticity and density of the underground medium. These response records are what we often call seismic records. The analysis of seismic records is used to infer geology. In addition, the model established by the method used in this paper shows that the relative error of thinning wells is relatively small, mainly concentrated around 1\%. Examination of the thinning wells of 45 wells shows that the model established basically has high accuracy. The research results of this paper have a guiding study about remaining distribution study place, tapping potentials, formulating reasonable development and adjustment plans, and improving recovery factors.

\section{Data Availability}

No data were used to support this study.

\section{Conflicts of Interest}

The authors declare that they have no conflicts of interest.

\section{Acknowledgments}

This work was supported by the National Natural Science Foundation of China (Grant No. 441972258) and the Science Foundation for Distinguished Young Scholars of Qinghai Province of China (Grant No. 2021-ZJ-980Q).

\section{References}

[1] K. Wawrzyniak-Guz, "Acoustic full waveforms as a bridge between seismic data and laboratory results in petrophysical interpretation," Acta Geophysica, vol. 64, no. 6, pp. 2356-2381, 2016.

[2] W.-F. Du, S.-P. Peng, and S.-Z. Shi, "Seismic interpretation of deep buried structure characteristics and its influence on coal mine safety[J]," Meitan Xuebao/journal of the China Coal Society, vol. 40, no. 3, pp. 640-645, 2015.

[3] O. A. Sanuade, "Seismic interpretation and petrophysical evaluation of SH field, Niger Delta," Journal of Petroleum Exploration and Production Technology, vol. 8, no. 1, pp. 5160, 2018.

[4] M. Bagheri and M. A. Riahi, "Modeling the facies of reservoir using seismic data with missing attributes by dissimilarity based classification," Journal of Earth Sciences, vol. 28, no. 4, pp. 703-708, 2017.

[5] C. L. Li, J. H. Zhang, H. K. Li, and C. Liu, "Application of new geological modeling technology in secondary development in Daqing oil field," IOP Conference Series: Earth and Environmental Science, vol. 40, no. 1, p. 012086, 2016. 
[6] H. Jiao and Z. Ding, "The seismic-geological comprehensive prediction method of the low permeability calcareous sandstone reservoir," Open Journal of Geology, vol. 6, no. 8, pp. 757-762, 2016.

[7] X. Zhang, K. Chen, and B. Ma, "The structural evolution characteristics of the sinian dengying formation gas reservoir and its controlling mechanism in the anyue gas field, Sichuan basin, China," Journal of Chengdu University of Technology (Science \& Technology Edition), vol. 45, no. 6, pp. 698-708, 2018.

[8] S. Du, P. Guan, and Y. Shi, "New fracturing criteria on low permeability sandstone reservoirs," Earth Science Frontiers, vol. 24, no. 2, pp. 257-264, 2017.

[9] C. Lin, S. Chen, and X. Zhang, "Probability constraint method based on multiple trend integration and its application in reservoir modeling," Acta Petrolei Sinica, vol. 36, no. 6, pp. 730-739, 2015.

[10] X. Chen, J. Zhang, and Y. Feng, "Remaining oil distribution features of heavy oil fault-block reservoir," Journal of Petrochemical Universities, vol. 30, no. 4, pp. 55-61, 2017.

[11] L. I. Yang, H. O. U. Jiagen, and L. I. Yongqiang, "Features and classified hierarchical modeling of carbonate fracture-cavity reservoirs," Petroleum Exploration and Development, vol. 43, no. 4, pp. 655-662, 2016.

[12] Z.-H. Ji and S.-G. Yan, "Properties of an improved Gabor wavelet transform and its applications to seismic signal processing and interpretation," Applied Geophysics, vol. 14, no. 4, pp. 529-542, 2017.

[13] Z. Lv, X. Li, and W. Li, "Virtual reality geographical interactive scene semantics research for immersive geography learning," Neurocomputing, vol. 254, pp. 71-78, 2017.

[14] Z. Lv, Y. Han, A. K. Singh, G. Manogaran, and H. Lv, "Trustworthiness in industrial IoT systems based on artificial intelligence," IEEE Transactions on Industrial Informatics, vol. 17, no. 2, p. 1, 2020.

[15] B. Tang, C. Wu, and X. Li, "A fast progressive 3D geological modeling method based on borehole data," Rock and Soil Mechanics, vol. 36, no. 12, pp. 3634-3638, 2015.

[16] X. Huang and H. Sun, "Numerical modeling of Gaussian beam propagation and diffraction in inhomogeneous media based on the complex eikonal equation," Acta Geophysica, vol. 66, no. 3, pp. 1-12, 2018.

[17] E. Mohamed, "The relation of artificial intelligence with internet of things: a survey," Journal of Cybersecurity and Information Management, vol. 1, no. 1, p. 30, 2020.

[18] R. Song, X. Qin, Y. Tao et al., "A semi-automatic method for 3D modeling and visualizing complex geological bodies," Bulletin of Engineering Geology and the Environment, vol. 78, no. 3, pp. 1371-1383, 2019.

[19] Z. Luo, J. Chen, and C. Xie, "Characteristics of mechanical response of surrounding rocks in deep hole pillarless retreating mining at deep stope," Science and Technology Review, vol. 33, no. 1, pp. 70-74, 2015.

[20] F. Guo, Z. Wu, and X. Li, "The 3D geological modeling of Xiangshan volcanic basin in Jiangxi Province," Geological Bulletin of China, vol. 37, no. 2, pp. 421-434, 2018.

[21] P. Shan and X. Lai, "Influence of CT scanning parameters on rock and soil images," Journal of Visual Communication and Image Representation, vol. 58, no. 1, pp. 642-650, 2019.

[22] P. Shan and X. Lai, "Mesoscopic structure PFC 2D model of soil rock mixture based on digital image," Journal of Visual Communication and Image Representation, vol. 58, pp. 407415, 2019.
[23] H. Qu, M. Pan, and X. Liu, "Urban 3-D geological modeling and its application to urbanization," Geological Bulletin of China, vol. 34, no. 7, pp. 1350-1358, 2015.

[24] T. O. Sonnenborg, D. Seifert, and J. C. Refsgaard, "Climate model uncertainty versus conceptual geological uncertainty in hydrological modeling," Hydrology and Earth System Sciences, vol. 19, no. 4, pp. 4353-4385, 2015.

[25] X. Z. Lyu, Z. H. Zhao, X. J. Wang, and W. M. Wang, "Study on the permeability of weakly cemented sandstones," Geofluids, vol. 2019, Article ID 8310128, 14 pages, 2019. 